\title{
A Novel Sleep Scoring Algorithm-Based Framework and Sleep Pattern Analysis Using Machine Learning Techniques
}

\author{
Sabyasachi Chakraborty, Inje University, South Korea \\ (iD) https://orcid.org/0000-0003-1344-296X \\ Satyabrata Aich, Inje University, South Korea \\ Hee-Cheol Kim, Inje University, South Korea
}

\begin{abstract}
Maintaining the suited amount of sleep is considered the prime component for maintaining a proper and adequate health condition. Often it has been observed that people having sleep inconsistency tend to jeopardize the health and appeal to many physiological and psychological disorders. To overcome such difficulties, it is often required to keep a requisite note of the duration and quality of sleep that one is having. This work defines an algorithm that can be utilized in smart wearables or mobile phones to perceive the duration of sleep and also to classify a particular instance as slept or awake on the basis of data fetched from the triaxial accelerometer. A comparative analysis was performed based on the results obtained from some previously developed algorithms, rule-based models, and machine learning models, and it was observed that the algorithm developed in the work outperformed the previously developed algorithms. Moreover, the algorithm developed in the work will very much define the scoring of sleep of an individual for maintaining a proper health balance.
\end{abstract}

\section{KEYWORDS}

Accelerometer, Algorithm, Classification, Machine Learning, Naïve Bayes Classifier, Random Forest Classifier, Sensors, Sleep Scoring, Voting Classifier

\section{INTRODUCTION}

The preeminence of having proper sleep is of the prime aspect for maintaining proper health. In the last two decades, the lifestyle of people has changed rapidly, either because of the customed busy life or because of the fine-tuned arrangement of living. People these days are quite reluctant to maintain or keep a check on the duration or the quality of sleep that they are having. Such kind of delinquency has often led to multiple sleep disorders and often lead to the stage of insomnia, apnea (Balakrishnan et al. (2005)) and a multitude of health intricacies. Therefore, maintaining a check on the quality, duration, and efficiency of sleep is an important routine that each individual needs to follow for seeking a proper health condition (Cheng and Mei, (2012)).

\section{DOI: 10.4018/IJSDA.2021070101}

This article, published as an Open Access article on April 23, 2021 in the gold Open Access journal, International Journal of System Dynamics Applications (converted to gold Open Access January 1, 2021), is distributed under the terms of the Creative Commons Attribution License (http://creativecommons.org/licenses/by/4.0/) which permits unrestricted use, distribution, and production in any medium, provided the author of the original work and original publication source are properly credited. 
Detection and identification of sleep have always been an active area of research and also the area has been acknowledged enough for different methods and processes to analyze the duration and efficiency of sleep quantitatively. As we know that polysomnography is considered as the "Paragon of Excellence" (Alickovic and Subasi, (2018)) for determining sleep quality and sleep stage classification, but the main complication behind polysomnography is the number of sensors, cables, and devices that are attached to the body and also the complete task needs to be carried out in a specific laboratory environment which even leads to basic distress to the subject who is carrying out the procedure. Therefore, a plethora of smartwatches and wearables are now available in the market for maintaining a proper check on the efficiency of the sleep and also to dictate terms of organizing the time to manage it as a whole.

For the wearable devices (Sathyanarayana et al. (2016) and Yeo et al. (2017)) to determine sleep efficiency and the sleep duration, the developers predominantly used the tri-axial accelerometer that is fetched from the wearable devices and determines a particular state or an epoch as a slept stage or awake stage. This classification of each epoch is computed to determine the efficiency and duration of the complete sleep cycle (Sadeh et al. (1994)). The purpose of this study is actually to develop a more generic algorithm for detecting and classifying every epoch of the data to be slept or awake. Also, the work tends to perform a comparative analysis between previous algorithms and also to perform a cross-technique analysis between rule-based methods and machine learning-based models. The method is completely based on statistical analysis which gives it the leap of behaving as a generic model, which can classify the epochs either for data that is being generated by the sensors present in a mobile phone or smart wearable devices.

The system defined in the work consumes the results from the algorithm as ground truths and further simplifies the complete system into an intelligent engine as it leverages machine learning algorithms to classify a particular epoch as slept or awake. The model is trained with statistical features (Yeo et al. (2017), and Aich et al. (2019)) that have been generated from the data and further trained on different algorithms for fetching higher recall and higher precision.

The paper is structured as follows: The next section shows the previous study and also depicts the types of methods developed for either representing the same problem or using the same sensor technology to address different problems. the third section typically is the one that addresses the complete development mechanism of the system and also explains the flow of control of the system starting from data extraction to the final model. This section also places forward the complete development mechanism of the algorithm and also examines the complexities associated with the algorithm for further research that may rise to concern while performing any further research on it. Moreover, in the particular segment, we also try to devise some feature engineering methods and feature extraction methods for feeding the data to the machine learning model in due course of the paper. The fourth section mainly plots the complete results obtained from the system and also to perform a comparative analysis between previous algorithms and the newly developed algorithm. the fifth section demonstrates the discussion on the developed system and, manages to argue upon the indifferences in other algorithms that developed the motivation for this particular work. The sixth and final section puts forward the conclusion.

\section{RELATED WORK}

Alickovic et al. (2018), suggested a method for sleep stage classification using a single-channel electroencephalogram and rotational support vector machine as the classification algorithm. The Signals that were considered for the system were from the $\mathrm{Pz}$ and $\mathrm{Oz}$ electrodes of the EEG device. The $\mathrm{Pz}$ and $\mathrm{Oz}$ electrode are the ones that extract signals from the occipital and inion region respectively and are highly specified for the analysis of the sleep disorders. The signals from the electrodes were further subjected to noise removal using the method of multiscale principal component analysis. Post denoising of the signals, some important features were extracted from the signals using discrete wavelet 
transform where signals of multiple frequency bands were fetched and their wavelet coefficients were calculated based on the statistical values of subbands. In the final segment, the features are passed on to a modified version of the support vector machine for classifying the sleep that is known as a rotational support vector machine. Almazaydeh et al. (2012), suggested a method for the detection of sleep apnea which signifies the pauses in breathing or low breathing during sleep. For the detection of the sleep apnea, the work suggested the usage of ECG signals from the subjects and classify the short duration sleep fragments as with or without apnea using support vector machines. The rotation-based ECG signals were taken which were further subjected to the feature's extraction process. Multiple features were extracted from the process, such as mean epoch, the standard deviation of the epoch and few variant based features. Post feature extraction, learning was performed on the data using support vector machines for classification purposes of apneic and non-apneic subjects. Chung et al. (2007), derived the method of classifying REM sleep and non-REM sleep by using the data from the respiration of three subjects. For identifications of the activity signal, load-cells were installed in the bed to identify the respiratory patterns of the subjects. For the validation of the REM and non-REM ground truths, the polysomnographic data is used to match up the correlation between respiratory patterns and polysomnographic data, which shows a high correlation with increasing respiratory activity.

Photoplethysmography based method proposed by Eyal et al. (2017), depicted some efficient way of sleep scoring. The proposed methodology uses an inter-beat interval based on heart rate variability. Sathyanarayana et al. (2016), compared multiple deep learning models such as CNN, RNN, LSTM, TB-LSTM and logistic regression using the data generated from the Actigraph Gt3X+for the detection and classification of sleep quality. It was found that the deep learning models performed extremely well in comparison to the probabilistic models. Tataraidze et al. (2015), performed a classification task by fetching the data from polysomnography study and classify the sleep, awake, REM, and nonREM. The primary aspect of the work is the addition of some different heuristics, which can increase the performance of the model. The features that were extracted from the signal were all cycle-based features, therefore the features generated from the signal are based on the power spectrum component of different frequency ranges. Post feature extraction, the features were subjected to the classifiers, namely. Random forest, bagging classifier, and KNN. It was observed that bagging classifiers with heuristics outperformed all other classifiers based on the accuracy of the model.

Some of the artificial intelligence techniques found to be effective and have been included in the research to give an idea to fellow researchers to choose the best classification approach for implementing in their research works. Majhi (2018), proposed a model using a sine cosine algorithmbased neural network (SCA-NN) for the classification of breast cancer and found that the approach was robust and provides better result compared to other classifiers. Panda (2019), proposed a method that used a hybrid classification method that includes neural network classifier based on radial basis function (RBF) based and instance selection based on distribution base balance(DBB) for the prediction of a software defect. It was found that the model is robust and effective and also validated using statistical tests. Singh et al. (2015) proposed a novel method that can identify the word-level script based on the features using texture and they have used several classifiers, among them MultiLayer perceptron was found to be the best classifier with an accuracy of $94.7 \%$.

Sanders et al. (2014), performed a completely new study for sleep stage classification using cross-frequency-coupling. The primary objective of the work was to develop a comparative analysis between cross-frequency coupling to traditional band power estimation and preferential frequency band information estimation. The features considered in the initial phase were the power from individual frames of one second from different frequency bands. For the initializing the CFC method, features were extracted which were calculated on each possible phase and sub-band combination. The extracted features were further used for classification using a bagging classifier of 200 tree ensembles to classify the sleep epochs as stage 1, stage 2, deep sleep and REM respectively. On the other hand, Zhang et al. (2017), demonstrated a method for sleep stage classification, that is, by enumerating the CW 
Doppler radar and using bagged trees algorithm for classification purposes. CW doppler radar is a noninvasive method for the detection of slight movement in the subject's body and also to determine respiratory and heartbeat movements which are further modulated into a signal and the displacement can be determined using the phase shift operator. Post feature extraction from the signals, a decision tree-based bagged classifier was used to classify the stages of sleep. Also, a similar kind of method with respect to the sleep stage detection using bio radiolocation was suggested by Tataraidze et al. (2016). The system tends to perform a noninvasive determination of sleep stages but considering the data from polysomnography as the ground truths. Following the feature extraction, the classification of the sleep stages was performed using an extreme gradient boosting method and a considerable result was obtained.

Yeo et al. (2017), described an automatic method for the detection of the sleep stages using coupled data from polysomnography and a tri-axial accelerometer. As polysomnography is considered as the "Paragon of Excellence" for determining the sleep stages of an individual, therefore it was used for fetching the ground truths of the sleep stages. Moreover, with the PSG data as actuals, triaxial accelerometer data was considered which supported being raw features of the system. The author intended to find out some features after properly denoising the accelerometer signals and therefore it was concluded that the classification that was performed on the signals gave a remarkable response, where the specificity for determining the REM and deep sleep as high as $90 \%$.

Sometimes the data used for developing a model become cumbersome because there is a lot of uncertainty is available on it and in some cases, it is necessary to look around the effect the surrounding data on the model. Some of the past works are mentioned here so that the fellow researchers will know how to compensate it while building the best model. Dutta (2017) proposed a method using a fuzzy set theory in which they have used interval-valued fuzzy sets (IVFS) and intuitionistic fuzzy set (IFS) for decision-making process based on the data that contains uncertainty and they found this method is effective in handling the uncertainty in the data. Ramadan and Altamimi (2017) proposed a method that can take care of the effect of the surrounding data while dealing with a different types of networks. They have used a hierarchal based fuzzy logic controller and found this method is effective. Elfouly et al. (2017) mentioned an efficient method for reporting the data that could be useful for the tracking of objects using wireless sensor networks. They have used different types of optimization method such as linear programming and swarm intelligence to achieve the desired objective.

The classification of some possible sleep disorders such as Cheyne stokes breathing and obstructive sleep apnea was performed by Suhas et al. (2006) using the ECG data and polysomnographic data. From the data generated using the ECG, two types of signals were derived namely, R-wave attenuation and heart rate variability, these features were further subjected to frequency domain analysis for the identification of OSA and CSR events. Sano and Picard (2014), performed a comparative study on sleep-wake classification using electroencephalogram data and wearable multi-modal sensor data Chouvarda et al. (2010). Moreover, in the work, some more data apart from EEG and accelerometer data were used such as skin conductance and skin temperature. And it was noted from the results that with only EEG data showed an accuracy of $83 \%$ and with only wearable sensor data, the accuracy was obtained to be $74 \%$ and was also subject dependent.

\section{METHODOLOGY}

\section{Data Collection}

The data that was used for the development and validation of the system was arranged from a single subject who wore the data for 30 nights. The nights were averaged to be around 6.5 to 8 hours Long. The interval of sleep considered the whole time of sleep onset to sleep offset. In the experiment performed, a triaxial accelerometer was worn by the subject, which was fetching out the data at the sampling rate of $26 \mathrm{~Hz}$. The accelerometer used for the purpose is Partron PW-250 as shown in Figure 
1(a), which is quite lightweight and plain sailing in the usage. The device weighs a mere 17 grams and is of the dimensions $49.10 \mathrm{~mm} \times 15.30 \mathrm{~mm} \times 10.9 \mathrm{~mm}$ which even makes it a perfect device to be worn during the time of sleep, to avoid any kind of discomfort to the subject. The accelerometer that is used in the study proves a sensitivity of $-8 \mathrm{~g}$ to $+8 \mathrm{~g}$, which can be considered for measuring a wide range of physical activities.

Figure 1(b) shows the strap or the band which holds itself responsible for strapping up the device on the subject's wrist. The dimension of each strap is around $80 \mathrm{~mm} \times 10 \mathrm{~mm}$. In Figure 1(c) we can observe the way the wearable device is been wore by the subject, this is the exact way in which the subject wore the device on the wrist and has undertaken the process of generating accelerometer data during the required hours of sleep.

In a further extension for performing the data collection procedure using the Partron PW-250 as shown in Figure 1(a), a smartphone-based method was also undertaken where an Android-based smartphone was leveraged to fetch the accelerometer data while the subject was lying on the bed and the phone was lying just aside his pillow. The prime aspect of leveraging the accelerometer sensors embedded into the android smartphone is that the sensors used in the phone have typically a higher sensitivity that is around $-4 \mathrm{~g}$ to $+4 \mathrm{~g}$ therefore even the slightest movements during sleep is captured by the device and also, we used an android application named AndroSensor for extracting the raw accelerometer data from the device. The app was directly sensing the data at a sampling rate of $4 \mathrm{~Hz}$.

\section{Proposed Algorithm}

\section{Description}

The Algorithm proposed and demonstrated in the work can be used for the scoring of sleep under normal conditions. The methodology for classifying each epoch of 60 seconds to either slept or awake has been determined by closely analyzing the patterns of the data that is been ingested to the system from a tri-axial accelerometer which is been wore by the subject during the experimental process. Pattern identification process from the data is based on the concept of time series analysis, where previous observations are considered using a rolling window for delivering a hypothesis for current or future predictions. In the previous work (Sadeh et al. (1994)) we have observed that for all activities only the data from the vertical axis i.e. $y$-axis is considered. But in this work, we have considered a resultant vector that emerges from the dot product of the three axes namely, $\mathrm{x}$-axis,

Figure 1. The device that was used for fetching the Accelerometer Data during sleeping: (a) The main device that encloses the sensor and also follows up with Bluetooth 3.0 connectivity for transferring raw data. (b) The Strap is used to affix the device. (c) Subject Wearing the band.

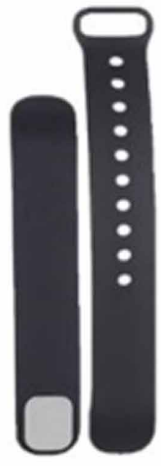

(b)

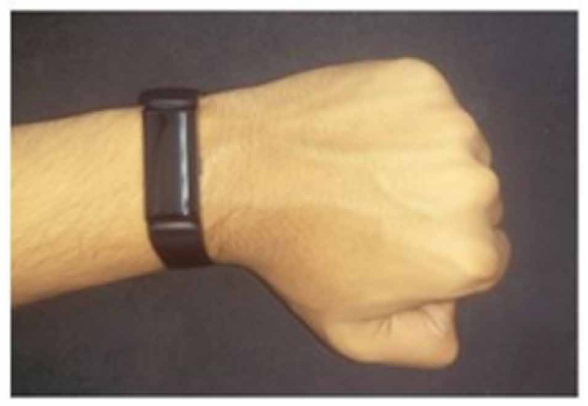

(c) 
$y$-axis, and z-axis and also the usage of resultant vectors helped us to identify a unified pattern with regard to the movement during sleep.

In the second module, we specify the implementation of a machine learning model to simulate the complete algorithm to be converted into a learning algorithm which in turn increases the robustness of the system. For the use of machine learning, we tend to perform extensive feature engineering on the signals to fetch out some features which relate highly to the output variable. Post feature engineering we stand the usage of three machine learning algorithms namely, random forest classifier, naïve Bayes classifier, and a voting classifier which performs average voting between the prediction probabilities of naïve Bayes classifier and random forest classifier with the weighted ratio of 1:3.

\section{System Process Outline}

The complete end to end pipeline of the system starting from the initial data ingestion to the comparative analysis is been depicted in Figure 2. In the initial step, the data is fetched to the system from the wearable device using an android application that provides RESTful Services for fetching the data directly. The data that is been generated by the accelerometer sensors in the wearable device is in the form of millig [ $1 \mathrm{millig}=0.0098 \mathrm{~m} / \mathrm{s} 2$ ], therefore conversion of the data from the extracted format to $\mathrm{m} / \mathrm{s} 2$ is very much required for further processing and to manage its implementation on the data using the following equations:

$\mathrm{y}=\frac{2 * \text { Sensitivity Range } * 1000}{2^{\text {Number of Bits }}}$

$\frac{\text { Value To Be Converted } * y}{1000} * 9.8 m / s^{2}$

where the sensitivity range of the device in millig tends to represent the hardware specification of the tri-axial accelerometer sensor that is been installed in the wearable device. For example, if the sensitivity of the triaxial accelerometer is $8 \mathrm{~g}$, therefore the numerator of the equation (i) is 16000 (Le et al. (2016)). In the denominator of the equation (i) we point out a variable known as the number of bits, which response to the memory size of the raw data received from the tri-axial accelerometer. In this work, the output size was 16 bits, therefore, the denominator of the equation becomes to 216 . In the second equation, the value that is to be converted is to be multiplied with the factor that we obtained from the equation (i) which gives out the hardware sensitivity of the device.

The data that is been received from the accelerometer contains a lot of noise and artifacts, therefore we apply a bandpass filter (Yimman et al. (2005)) for removing all the signals above $10 \mathrm{~Hz}$ and Lower than $0.88 \mathrm{~Hz}$ because of our primary task of fetching the proper signals is satisfied by the range of $0.88-10 \mathrm{~Hz}$. Moreover, the tri-axial accelerometer registers the data at a sampling rate of $26 \mathrm{~Hz}$, therefore in the preprocessing step, the downsampling of data has to be performed to $4 \mathrm{~Hz}$ which is a primary requirement for the model. In the third step, we perform a rolling and aggregation task for considering each epoch to be of 60 Seconds. This process is performed to relate up to a single epoch as a module or can be more specifically considered as a sleep segment.

The fourth segment is considered to be the driving unit of the complete system, as this segment classifies every epoch passed across it as slept or awake. The segment performs variated statistical operations to identify patterns in the subject's sleep and also identifies the exact movement of the subject at a continuous rate. In a more specific way, the first stage determines the type of sleep the subject is having or the time it takes for the subject to transition from awake to sleep. As the transition time is certainly always different for different people, therefore, it is always advised to mark the transition time from the data itself without putting in any static hypothetical value. In 
Figure 2. This Figure depicts the complete end to end the pipeline of the system

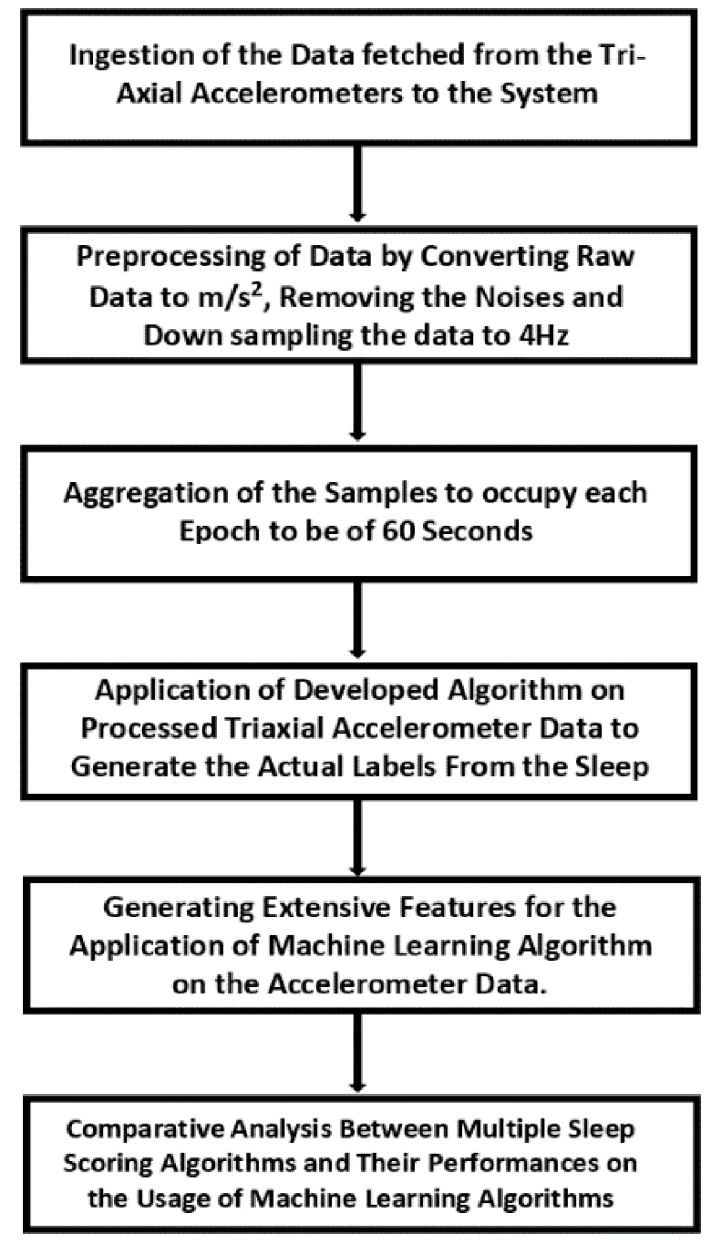

the second segment, we identify the rolling difference threshold where we identify the slightest movement continuously between five immediate epochs by rolling a window. The method helps us to correlate with the first segment to identify the slightest vibrations. Therefore, by performing the above-mentioned processes, we can easily identify if a person is slept or awake.

The fifth segment is one of the most important steps for applying a machine learning model, as this step deals with extensive feature engineering on the processed tri-axial accelerometer data. The features that were used were basically on either the relation of two axes at a particular epoch or it is either on the entropy gain from the axes by neglecting the artifacts or self-transition features where each value scans all its previous value and calculates the mean or standard deviation or variance and fetches a single value in association with that particular epoch. The feature engineering provides us with information that can be found from the interaction of variables and performing statistical operations on the axes. In the study performed (Alickovic and Subasi (2018), Yeo et al.(2017 and Galland et al.(20))) we can also see that the features considered are also of the same type, either interaction-based or the self-transition features. Moreover, with regard to the evaluation metric for deciding the performance of the algorithm, we tend to use precision and recall of both classes namely slept and awake, as the hypothesis suggests that the precision of the majority class should be high 
i.e. slept class and the recall of the minority class should be high i.e. awake class. In the last and the final segment, we perform a thorough comparative analysis of the commonly used algorithm known as Sadeh (Sadeh et al. (1994)) and the proposed algorithm on multiple machine learning models to perform a quantitative test of both the algorithms to derive the accuracy and efficiency.

\section{Algorithm Model}

The Algorithm proposed in the work works on the pre-processed data that is been generated by the preprocessing module of the system. All through the algorithm can even work with the raw data coming directly from the accelerometer, but it is advisable to operate using the processed data which are in the unit of $\mathrm{m} / \mathrm{s} 2$, having a sampling rate of $4 \mathrm{~Hz}$ and all the signals must be in the range of $0.88-10 \mathrm{~Hz}$. Moreover, the complete algorithm is based on time series motive to analyze the pattern and trends of sleep that the subject undertakes and post-analysis we perform a variance check to the movement threshold of the subject. The movement threshold helps us to identify the behaviors of the subject concerning the type of sleep that prevails with him. The following steps depicted below helps us to identify the specifics of the algorithm in a better way.

Step 1: To determine the resultant vector at each epoch using the below-mentioned equation:

$$
\overrightarrow{\mathrm{R}}=\sqrt{\mathrm{x}^{2}+\mathrm{y}^{2}+\mathrm{z}^{2}}
$$

Explanation 1: In some previous work we have observed (Sadeh et al. (1994)) that the calculation of sleep scoring is often done by considering the y-axis or the vertical axis vector. But in this case, we try to consider the data of all the axis and to find out the resultant that leads us to the overall or resultant movement in all directions.

Step 2: To determine the mean of the resultant vector:

$$
A=\frac{1}{n} * \sum_{i=1}^{n} R_{i}
$$

Explanation 2: The mean of the resultant vector is utilized to create a sense of low-pass filter where the data rating of the vertical axis more than the mean of the resultant vector is capped at the mean value itself.

Step 3: To cap all the values of the vertical axis or the y-axis more than the mean of resultant vector to the mean itself at each epoch:

$$
X=\operatorname{minimum}\left(\text { Axis }_{y}, A\right)
$$

where axis-y is the array of vertical axis values and a is the resultant vector mean.

Explanation 3: Capping off all the values is performed on the y-axis to determine the maximum value for the signal to be the mean of resultant vector and neglecting all the values above the mean of the resultant vector. Removing the data above the threshold limit of the vertical axis or the $y$-axis that is above the mean of resultant vector allows focusing more on the data that is been captured while sleeping or lying down on the bed and neglecting the data of higher value to fetch a basic pattern at a cold level of human activity. 
Step 4: Applying the rolling vibration threshold to all the three axes

Explanation 4: Rolling vibration threshold is considered to be the core unit of the algorithm, as it processes a few delicate operations on the data that is passed to it. The architecture of the rolling vibration threshold is being shown in Figure 3. In the very first step, we tend to append four zeroes to the list of data because of two reasons that are, the window size for the generation of the convolving subsets is 5 and the system is defined by balancing on a prepended list as we need to provide the rolling vibration threshold for the very first element. Therefore, for the calculation of the rolling vibration threshold, we added four zeroes at the beginning of the list so that the first element becomes the fifth element of the convolving subset and further tasks can be performed chronologically on every item on the list. In figure 3 , the fifth element that is X5 of the array is considered for demonstrating the calculations to derive the rolling vibration threshold value that is $\mathrm{Z} 5$. As the rolling window or convolving window size is equal to 5 , therefore, we need to consider in total 4 more elements including $\mathrm{X} 5$ that comes before it. Now in the second segment, as we have generated the subset that is $\mathrm{X} 1, \mathrm{X} 2, \mathrm{X} 3, \mathrm{X} 4$, and $\mathrm{X} 5$, we apply a subtraction function on consecutive elements to fetch the difference vectors which are Y1, Y2, Y3, and Y4. Post deriving the difference vectors, the mean of those vectors is calculated and $\mathrm{Z} 5$ is derived in the process which directly

Figure 3. The flow of Control for the Rolling Vibration Threshold

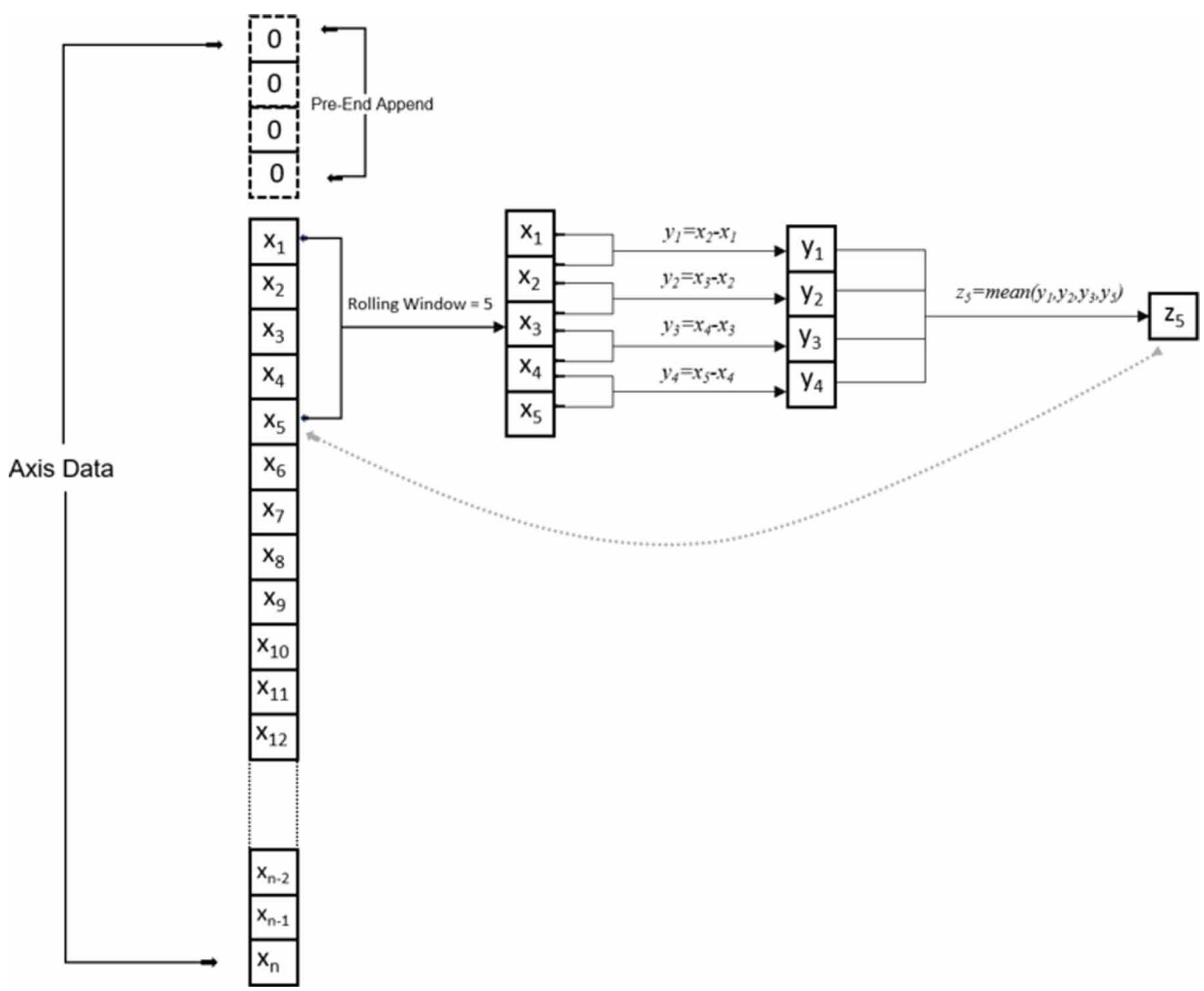


relates to X5. The process performed above allows us to find the convolved or the recurrent vibrations across the previous four epochs concerning a particular epoch and also presumes the threshold value for a subject to determine whether the subject is slept or awake. This particular process is performed for all three axes individually.

Step 5: To find the Resultant Rolling Vibration Threshold

$$
\vec{V}=\sqrt{Z_{x-a x i s}^{2}+Z_{y-a x i s}^{2}+Z_{z-a x i s}^{2}}
$$

Explanation 5: The resultant rolling vibration threshold, allows us to capture the vibration occurred during the sleeping time of the subject in all dimensions. This step also resolves the discrepancy that may occur by considering a single axis where for a particular instance the vibrations maybe only on a single axis which is much unlikely.

Step 6: To find the standard deviation of the resultant rolling vibration threshold:

$B=\sqrt{\frac{1}{n} \sum_{i=1}^{n}\left(V_{i}-\mu\right)^{2}}$

where $\mu$ is the mean of $\mathrm{V}$.

Explanation 6: Here the standard deviation of the resultant rolling vibration threshold is calculated to compare with the resultant rolling vibration threshold vector to allocate every epoch with a label that is either slept or awake. The standard deviation helps to realize the imminent deviation of the mean from every value of the epoch.

Step 7: To perform the comparison between the standard deviation of resultant rolling vibration threshold and each value of resultant vector $\mathrm{V}$ using the following segment:

if $\mathrm{Vi}<\mathrm{B}$, then Class $=$ "Slept"

else, Class $=$ "Awake"

Explanation 7: In this case, we have mentioned that if a particular epoch of the resultant rolling vibration threshold is less than its standard deviation value then the epoch is classified as slept else it must be considered as awake.

\section{Feature Engineering and Feature Selection}

In the previous segment, we have used the data from the triaxial accelerometer for the calculation of sleep scoring as slept or awake. So, for leveraging some machine learning techniques in the following segments to train the machine with the available patterns of data from the tri-axial accelerometer to predict the sleep scoring without moving into the much extensive algorithmic calculation, we perform feature engineering on the data. Also, there lies another reason behind the feature engineering task that we perform is the avoidance of the machine getting overfitted with the data directly from the accelerometer because the data is the primary medium of generating the sleep scores that are slept or awake. Therefore, we used some statistical methods, for generating the right number of features for our model and the features that are been developed and also the features must be of adequate use 
which can be confirmed as a reliable source that may possess exact patterns from the accelerometer data. (Yeo et al. (2017) and Aich et al. (2018))

Table 1 shows all the six different features that are required for the implementation of the learning algorithm for classification of the accelerometer data as slept or awake (Kwon et al. (2014)). The features listed above in Table 1 maintain a proper relationship with the type of data we are using and also are statistically significant.

\section{Machine Learning Implementation and Performance Estimation}

In recent years, we have seen the way machine learning has evolved and the way it disrupted the technological community. With the advent of machine learning, all the sectors present day are roped up in the science behind the way it can leverage the persona of automation and intelligence. In the health care industry, we have clearly observed the way machine learning and artificial intelligence is being utilized for the development of some really intelligent models that have laid the foundation of smart health care and are being used in the present day by a lot of human beings (Acampora et al. (2013)). This machine learning model described in the work specifically team up to develop a system which in turn can relate to predicting the score of activity during a sleeping hour that is either slept or awake. Usage of machine learning algorithms tends to define a much efficient way of scoring the sleeping activity as the primary model is trained with the data, which has got the true labels generated from the scoring algorithm. Therefore, a further run of the algorithm for the testing data or the data that would be getting into the system in due course of time will not be required to be carried upon.

Table 1. Descriptions of all the features. Each feature was calculated for the $x$-axis, $y$-axis, and z-axis. Therefore, a total of 18 features were calculated.

\begin{tabular}{|c|c|c|}
\hline Feature & Equation & Description \\
\hline Correlation & $\operatorname{Corr}(x, y)=\frac{1}{N-1} \sum_{n=1}^{N} \frac{\left(x_{i}-\bar{x}\right)\left(y_{i}-\bar{y}\right)}{\operatorname{std}(x)^{*} \operatorname{std}(y}$ & $\begin{array}{l}\text { The Correlation Coefficient Between Two } \\
\text { Accelerometer Signals. }\end{array}$ \\
\hline Kurtosis & $\operatorname{Kurt}(x)=\frac{E\left[(x-\bar{x})^{4}\right]}{\operatorname{std}(x)^{2}}$ & $\begin{array}{l}\text { Kurtosis is known to be the Peakedness of a } \\
\text { Signal. The Fourth Central Moment and Standard } \\
\text { Deviation is used to compute it. }\end{array}$ \\
\hline Skewness & $\operatorname{Skew}(x)=E \| \frac{x-\bar{x}}{\operatorname{std}(x)}$ & $\begin{array}{l}\text { Skewness of the Distribution of the Accelerometer } \\
\text { Data. }\end{array}$ \\
\hline Entropy & $\operatorname{Ent}(x)=-\sum_{k} p_{x}(k) \ln \left(p_{x}(k)\right) 1$ & $\begin{array}{l}\text { The Probability Mass Function of Accelerometer } \\
\text { Data. }\end{array}$ \\
\hline Crest Factor & $\operatorname{Crest}(x)=\frac{\max (x(n))}{\sqrt{\frac{1}{N-1} \sum_{n=1}^{N} a(n)^{2}}}$ & $\begin{array}{l}\text { It shows the Signal Impulsiveness with the } \\
\text { maximum accelerometer Value. }\end{array}$ \\
\hline $\begin{array}{l}\text { Discrete } \\
\text { Fourier } \\
\text { Transform }\end{array}$ & $D F T(x)=\sum_{n=0}^{N-1} x_{n} \cdot e^{-\frac{2 \pi i}{N} K n}$ & $\begin{array}{l}\text { It converts a sequence of finite equally spaced } \\
\text { samples to an equally spaced sample of discrete } \\
\text { time flourier transform. }\end{array}$ \\
\hline
\end{tabular}


The machine learning algorithms that are used in the work are random forest classifier, naïve Bayes classifier (Netti and Radhika (2015)), and voting classifier (Hong et al. (2007)) based on the average voting from the prediction probabilities of naïve Bayes classifier and random forest classifier. The use of only these three classifiers has got onto some explanation related to the type of data and features that we have used. Firstly, as the data that we have in our model or to be more specifically the engineered features are in a continuous domain with regard to the binary classification that we intended to perform. In such kind of scenario where we have a classification problem but the data that we have in the continuous domain, the most preferred way is leveraging probabilistic measures for the classification of the data and thus we ended up using naïve Bayes classifier. But as we know, all our labels have the raw signature of a three axes accelerometer and also the features derived in the process are also derived from the three axes. So, there is always a probability that the features that we tend to use will have a high correlation and moreover scaling the values in the context will also not help the algorithm to overcome the correlation-based issue. Therefore, at times, the usage of naïve Bayes classifier can be termed as a perfect algorithm for such kind of classification where we have features that belongs to the continuous domain but if the features have a high correlation and covariance the naïve Bayes classifier itself is typically not a better choice.

The second algorithm that we have taken up for the training of our machine learning model is the random forest classifier which often performs very well. But the major reason for considering a tree-based ensemble algorithm for the particular type of classification is that it deals perfectly with the features which have a high correlation and covariance. But there also remains a slight hiccup in using random forest classifiers for the data which are in a continuous domain i.e. our tri-axial accelerometer data because random forest classifier has shown exemplary performance in dealing with categorical data.

The third algorithm that we have considered is a voting classifier that performs soft or average voting against the prediction probabilities obtained from the naïve Bayes classifier and random forest classifier. as discussed in earlier, that the features that we have engineered from the raw tri-axial accelerometer data is perfectly fixed range continuous and moreover as the features are engineered only by considering the three axes of the accelerometer, therefore values engineered from a single domain will actually have a high correlation and covariance respectively. Therefore, for dealing with the continuous data, we tend to use naïve Bayes classifier and for dealing with regard to the high collinearity of data, we use random forest classifiers. Post-training the machine learning model on both the algorithms, the prediction probabilities of both the algorithms of both the classes are fetched and an average or soft voting is done on the slept class and the evaluation was done.

The hypothesis for this particular machine learning problem points out the fact that during the sleep duration it is more important to catch the epochs where the subject was awake or he was having a restless sleep (Blahut (1974)). So, identifying the epochs were the subject is actually awake during the sleep interval with higher accuracy is very much important. Therefore, the evaluation metric that was used for validating the stability and the accuracy of the model was performed by using recall, precision, and f1-score of both the classes. The recall specifically determines that in a particular class, the ratio of correctly predicted observations to all the samples of that particular class, therefore it is done on the basis of class level and the values of recall of both the class must be optimized but specifically seeking more care for a higher recall for the awake class, as suggested by our hypothesis. Precision is basically considered as that in a particular class either slept or awake, the ratio between the number of correctly predicted observations of a particular class with all the observation that are predicted as that particular class which is correctly predicted or wrongly predicted. F1-Score is the weighted average of both precision and recall and therefore takes a leap over all the wrongly predicted samples that are the false positives and false negatives.

In the system developed in the work, we consider all the epochs where the subject slept and we correctly predicted as slept are the true positives and all the epochs where the subject slept and we predicted as awake were considered as the false negatives (Chen and Tang, (2004)). For the second 
class or the class where the subject was actually awake, the true negatives were such observations where the subject was awake and we correctly predicted it as awake and the false positives are the one where the subject was actually awake and we predicted as slept. Based upon our hypothesis we know that we are performing the machine learning on the data captured during the period when the subject is sleeping, therefore, we have a bit lower number of epochs for awake class as compared to the slept class which leads to an imbalanced classification (Xiaoying and Sheng et al. (2012)) and therefore we tend to increase the recall of the awake class or increase the true negatives by reducing the false positives in a way to increase the precision of the slept class.

The data obtained and used for the work was for 30 Days and had 12652 epochs of sleep data of a subject and regarding the data distribution for training and validation, the split was of 70:30 ratio where both the parts were considered on a random day-based sequence where data of 21 random days i.e. 8956 epochs were used for the training purpose and data of 9 random days i.e. 3696 epochs were considered for the validation purpose.

\section{RESULTS}

The algorithm developed in the study aimed to provide some very sound results on the basis of the actual sleeping activity that was undertaken by the subject. Also, the machine learning model that was developed in the work provided with results by prompting an average accuracy of $96 \%$ over 5 -fold cross-validation and also showcased a constant tendency in the multiple random folds of the data of different days. Moreover, with accordance to the hypothesis that was stated, rules in the fact that the recall of the minority class that is awake must be higher and precision of the majority class that is slept must be higher which dictates the hypothesis is such a way that the system absolutely needs to detect more of awake states carefully and correctly while the detection of sleep states should be more precise is being held up by the system.

In Figure 4 the resultant vibration of all the three axes of a single night has been plotted after running it to through the rolling vibration threshold module. The average of the resultant vibration as depicted in Figure 4, comes to be at 5.34 on the vertical axis of the plot. Therefore, all the epochs having value of less than 5.34 will be considered as the state of sleep and more than 5.34 will be considered as the state of awake.

Table 2 follows a quantitative analysis of the performance of the machine learning algorithms on the results of the algorithm based on 5-fold cross-validation. For the analysis purpose, we can begin recapitulating the hypothesis of the problem which stays as the precision of the majority class

Figure 4. The Resultant Vibration Plot

Vibration Identification Plot

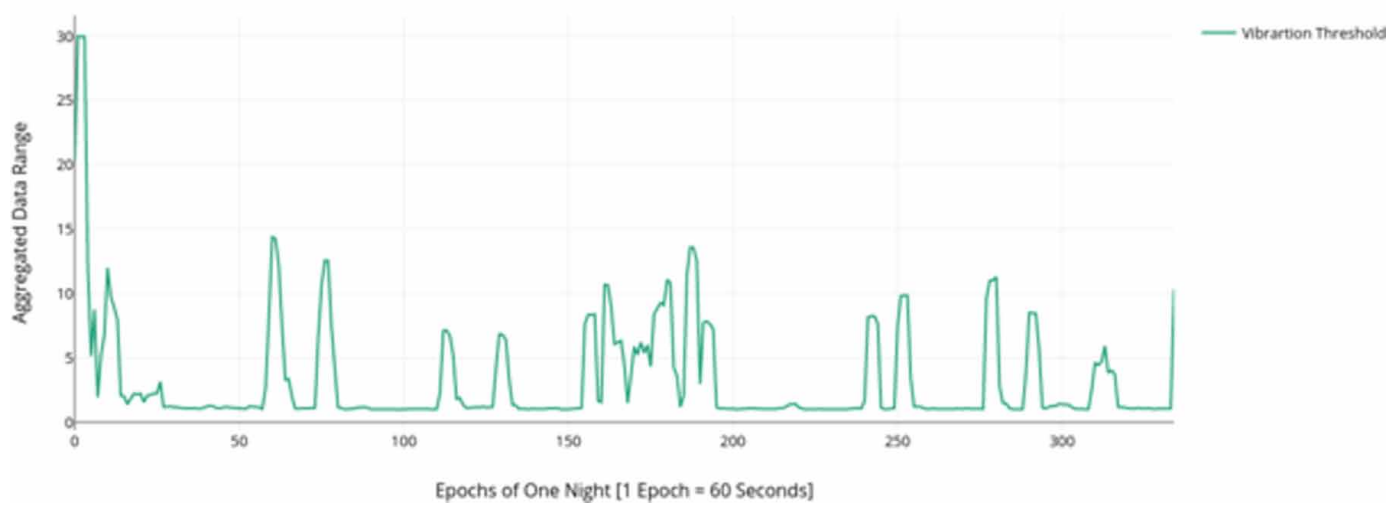


Table 2. Performances of all the three algorithms with a 5-Fold Cross-Validation

(a) Random Forest Classifier

\begin{tabular}{|c|c|c|c|c|c|c|}
\hline \multicolumn{7}{|c|}{ (a) Random Forest Classifier } \\
\hline Metric & 1st Fold & 2nd Fold & 3rd Fold & 4th Fold & 5th Fold & Average \\
\hline Model Accuracy & 0.95319 & 0.94291 & 0.92127 & 0.95400 & 0.96050 & 0.94432 \\
\hline Recall Slept & 0.98160 & 0.95650 & 0.95734 & 0.98034 & 0.96905 & 0.96855 \\
\hline Recall Awake & 0.90115 & 0.91801 & 0.85517 & 0.90575 & 0.94483 & 0.90989 \\
\hline Precision Slept & 0.94790 & 0.95530 & 0.92373 & 0.95014 & 0.96986 & 0.95185 \\
\hline Precision Awake & 0.96393 & 0.92012 & 0.91626 & 0.96176 & 0.94338 & 0.94051 \\
\hline F1-Score Slept & 0.96445 & 0.95590 & 0.94023 & 0.96501 & 0.96946 & 0.96007 \\
\hline F1-Score Awake & 0.93149 & 0.91906 & 0.88466 & 0.93291 & 0.94410 & 0.92475 \\
\hline \multicolumn{7}{|c|}{ (b) Naïve Bayes Classifier } \\
\hline Metric & 1st Fold & 2nd Fold & 3rd Fold & 4th Fold & 5th Fold & Average \\
\hline Model Accuracy & 0.93155 & 0.91694 & 0.94995 & 0.93209 & 0.90990 & 0.92808 \\
\hline Recall Slept & 0.92430 & 0.95399 & 0.97574 & 0.96989 & 0.96027 & 0.95684 \\
\hline Recall Awake & 0.94483 & 0.84904 & 0.90268 & 0.86284 & 0.81762 & 0.87540 \\
\hline Precision Slept & 0.96845 & 0.92050 & 0.94837 & 0.92834 & 0.90608 & 0.93435 \\
\hline Precision Awake & 0.87199 & 0.90969 & 0.95307 & 0.93990 & 0.91824 & 0.91858 \\
\hline F1-Score Slept & 0.94586 & 0.93695 & 0.96186 & 0.94866 & 0.93239 & 0.94514 \\
\hline F1-Score Awake & 0.90695 & 0.87832 & 0.92719 & 0.89972 & 0.86502 & 0.89544 \\
\hline \multicolumn{7}{|c|}{ (c) Voting Classifier (Random Forest and Naïve Bayes) } \\
\hline Metric & 1st Fold & 2nd Fold & 3rd Fold & 4th Fold & 5th Fold & Average \\
\hline Model Accuracy & 0.97700 & 0.95671 & 0.87852 & 0.91288 & 0.75676 & 0.93626 \\
\hline Recall Slept & 0.97951 & 0.98536 & 0.89126 & 0.95358 & 0.86198 & 0.95759 \\
\hline Recall Awake & 0.97241 & 0.90421 & 0.85517 & 0.83831 & 0.56398 & 0.89716 \\
\hline Precision Slept & 0.98486 & 0.94962 & 0.91853 & 0.91530 & 0.78365 & 0.94468 \\
\hline Precision Awake & 0.96282 & 0.97119 & 0.81105 & 0.90788 & 0.69043 & 0.92225 \\
\hline F1-Score Slept & 0.98218 & 0.96716 & 0.90469 & 0.93404 & 0.82095 & 0.95092 \\
\hline F1-Score Awake & 0.96759 & 0.93651 & 0.83253 & 0.87171 & 0.62084 & 0.90897 \\
\hline
\end{tabular}

i.e. slept must be high and the recall of the minority class i.e. awake must be high. Also, in terms of the usage of machine learning algorithms, it is always recommended to perform cross-validation to check the stability of the model and how it performs on the data from different days which appear on the test set and also how consistently it reacts to the changes in patterns if any across the time. Now, in the first algorithm that is the random forest classifier we can see that the accuracy is very slightly variable and ranges from $92 \%$ to $96 \%$ upon all the folds. Moreover, the recall of the awake stage is also relatively high as compared to the results shown in other algorithms and the precision of the slept stage is also high as compared to the other algorithms. For the case of naïve Bayes classifier, the accuracy ranges from $90 \%$ to $94 \%$ which is actually quite acceptable but the major snag of using this algorithm is that the establishment of the hypothesis test of other algorithms is fairly better than the naïve Bayes classifier. The third machine learning algorithm that has been used for the performance test was the voting classifier which performed an average voting between the random forest classifier and naïve Bayes classifier showed a pretty healthy performance in some of the data 
folds by scoring a $97 \%$ accuracy but in some with $75 \%$ accuracy which is not consistent enough. So, a voting classifier is not a very suitable one for the case that has been discussed in the work. Therefore, the best classifier for the system would be the random forest classifier which maintained a proper consistency of accuracy on all the data folds and also established a check with the proposed hypothesis theory. The confusion matrix that describes the best model has been shown in Figure 5 which holds and accuracy of $96.04 \%$.

\section{DISCUSSION}

The work that is specified in the paper demonstrated a novel method for determining sleep scoring based on the accelerometer data that is generated from a wearable device as well as a smartphone with high accelerometer sensitivity. The previously developed algorithms such as Sadeh and Cole (Sadeh et al. (1994) and Cole et al. (1992))were used for the purpose of scoring the sleep as either awake or slept based on the accelerometer data that was generated from subjects under multiple conditions. Also, conventional sleep scoring algorithms even showed substantial authority and confidence in determining the epochs to be slept or awake. But as demonstrated in the developed algorithm for this particular study, certain situations were taken into consideration for defining the algorithm to be more generic in terms of device independence and also not considering the age of the subject whose data we are extracting. Moreover, the developed algorithm teams up to be more dynamic in terms of fetching the factual properties of the data rather than going into some static values that need to be changed or modified in the case to case basis.

In the conventional algorithms like Sadeh (Sadeh et al. (1994)) it was observed that the study was performed under specific conditions and also the subjects were chosen based upon the age metric. The Sadeh sleep scoring algorithm that was a known conventional method was validated on the data from subjects who were 1 year old where the accelerometer was worn on the ankle of the subject and the data was being fetched. Also, Sadeh et al. (1994) performed the study on slightly older children and also for the adolescents where the triaxial accelerometer was worn on the non-dominance wrist. But, the study performed by Sadeh et al. 1994 suggested the use different algorithms for the children of different age groups which in turn again brings the pace of setting up static variables or static constraints for scoring the sleep of a particular subject. Moreover, Alickovic et al. (2018) also utilized the EEG data for the classification of varied sleep stages with accordance to the polysomnography data that was used for recovering the ground truths of each and every epoch. But scoring of sleep based

Figure 5. Confusion Matrix of Best Performing Model

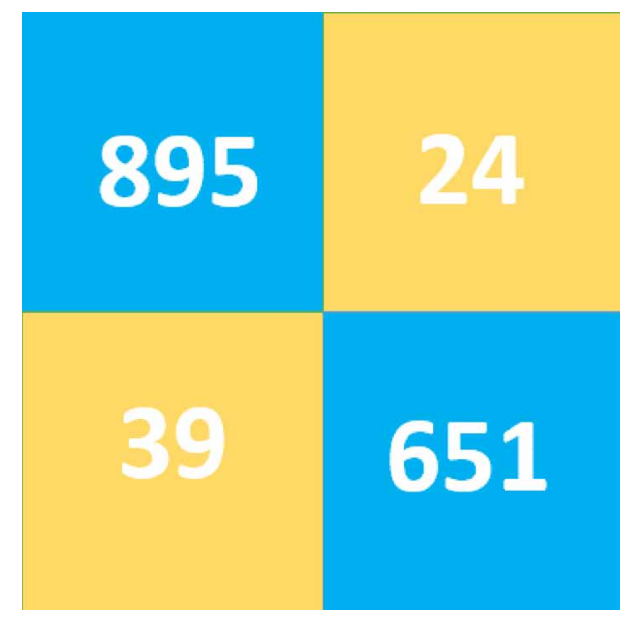


on EEG Data scores the sleep on the basis of the spikes that are generated on the nebular region of the brain and can lead to an inconsistency during the Non-REM stages and let out the segmentation of the data as awake for that particular instance of time.

The algorithm that is developed in the work maintains the fact of considering the automated preprocessing of the data that is generated by the accelerometer. Also, as we know multiple devices have different kinds of specifications for the sensor that is been used in the development of the wearable device or smartphone and also multiple devices have variated range of sampling frequency in which the data is fetched from the tri-axial accelerometer. To serve the vision of the development of the generic algorithm which fetches the data from the preprocessing segment of the system which in prior performs a dynamic conversion on the basis of the requirement that has been maintained for the structural working of the algorithm. Also, the major significance of using the algorithm developed in this work marks upon the fact of detecting the merest vibration of the subject and seeking the pattern of movements that are carried out by the subject during the complete sleep duration and firmly deriving the class of an epoch either slept or awake based upon the resultant threshold that has been calculated.

For a sleep scoring algorithm (Balakrishnan et al. (2005), Cheng and Mei (2012) and Yeo et al. (2017)) to be defined as a perfect one it is always paramount and necessary that we consider the sleeping patterns of the individuals whose data we are capturing. Now, we know that each and every human being has their own way of sleeping and have a unique way of how they spend their sleeping time with slight movements or vibrations as discussed previously in the work. Moreover, in a real scenario, it has also been observed that the devices that just use accelerometer data for scoring the sleep tend to get the information from the user regarding their nonactive hours and try to predict the movements during that particular interval of time by varying a threshold. But if the Sleep Scoring is to be done in a more appropriate way, then it is always advised to detect the segment of sleep data automatically from the whole activity data that has been recorded across a day. Therefore, the algorithm that has been proposed and developed in the study gives around a generic methodology to leverage machine learning algorithms to identify the particular sleep segment out of the overall daily activity.

The machine learning model that has been developed performs with an average accuracy of $96.04 \%$ correct predictions based upon the features that were engineered on the basis of statistical significance and previously performed research. The usage of the algorithm in the work has the prime aspect of supporting sleep scoring even in the nonactive hours of the subject where we can see the segment which observes the rolling vibration threshold, as it has been defined that for a window of 5 epochs continuously, the system manages the difference between consecutive elements and further calculates the mean of that particular window. The mean that was calculated in the segment allows us to define the property of stagnation of the subject in accordance with the accelerometer activity. Therefore, to get the machine to learn regarding the properties, of the subject's movement pattern during the sleeping interval and also to differentiate between the sleeping activity and daily activity, the system defines a machine learning model that cures the issue of static allocation of interval. The usage of multiple machine learning models on the data leads to the selection of random forest classifiers which gave an average accuracy of $94.4 \%$ and an average recall of $93 \%$ which was the basis of proving the hypothesis of the system.

\section{CONCLUSION}

The work demonstrated in the paper, leveraged low-cost and hassle-free wearable devices to sense the motion details and patterns of the subject during the sleeping interval to score each and every epoch to be either slept or awake which can, in turn, define the efficiency of the sleep. The paramount significance of using this algorithm for scoring the accelerometer activity during the sleeping interval is that the algorithm in the initial stages defines the patterns of the subject and restricts a mechanism of scoring based on the patterns which may differ from person to person. Also, the work demonstrated the usage of a machine learning model that prompted the usage of some statistically significant features 
which in turn demonstrated an optimum performance with the best accuracy of $96.04 \%$ regarding the scoring mechanism. The scoring mechanism in turn developed will be very much useful for managing the sleep sequences by an individual for maintaining balanced and adequate health conditions.

\section{ACKNOWLEDGMENT}

This research was supported by the Ministry of Trade, Industry, and Energy (MOTIE), Korea, through the Education program for Creative and Industrial Convergence (Grant number N0000717). 


\section{REFERENCES}

Acampora, G., Cook, D. J., Rashidi, P., \& Vasilakos, A. V. (2013). A survey on ambient intelligence in healthcare. Proceedings of the IEEE, 101(12), 2470-2494. doi:10.1109/JPROC.2013.2262913 PMID:24431472

Aich, S., Chakraborty, S., Sim, J. S., Jang, D. J., \& Kim, H. C. (2019). The Design of an Automated System for the Analysis of the Activity and Emotional Patterns of Dogs with Wearable Sensors Using Machine Learning. Applied Sciences (Basel, Switzerland), 9(22), 4938. doi:10.3390/app9224938

Aich, S., Pradhan, P. M., Park, J., Sethi, N., Vathsa, V. S. S., \& Kim, H. C. (2018). A validation study of freezing of gait (FoG) detection and machine-learning-based FoG prediction using estimated gait characteristics with a wearable accelerometer. Sensors (Basel), 18(10), 3287. doi:10.3390/s18103287 PMID:30274340

Alickovic, E., \& Subasi, A. (2018). Ensemble SVM method for automatic sleep stage classification. IEEE Transactions on Instrumentation and Measurement, 67(6), 1258-1265. doi:10.1109/TIM.2018.2799059

Almazaydeh, L., Elleithy, K., \& Faezipour, M. (2012, August). Obstructive sleep apnea detection using SVMbased classification of ECG signal features. In 2012 annual international conference of the IEEE engineering in medicine and biology society (pp. 4938-4941). IEEE.

Balakrishnan, G., Burli, D., Behbehani, K., Burk, J. R., \& Lucas, E. A. (2006, January). Comparison of a sleep quality index between normal and obstructive sleep apnea patients. In 2005 IEEE Engineering in Medicine and Biology 27th Annual Conference (pp. 1154-1157). IEEE.

Chen, L., \& Tang, H. L. (2004). Improved computation of beliefs based on confusion matrix for combining multiple classifiers. Electronics Letters, 40(4), 238-239. doi:10.1049/el:20040176

Cheng, S. P., \& Mei, H. (2012, September). A personalized sleep quality assessment mechanism based on sleep pattern analysis. In 2012 Third International Conference on Innovations in Bio-Inspired Computing and Applications (pp. 133-138). IEEE. doi:10.1109/IBICA.2012.55

Chouvarda, I., Rosso, V., Mendez, M. O., Bianchi, A. M., Parrino, L., Grassi, A., \& Maglaveras, N. et al. (2010). EEG Complexity during Sleep: on the effect of micro and macro sleep structure. In 2010 Annual International Conference of the IEEE Engineering in Medicine and Biology (pp. 5959-5962). IEEE.

Chung, G. S., Choi, B. H., Kim, K. K., Lim, Y. G., Choi, J. W., Jeong, D. U., \& Park, K. S. (2007, November). Rem sleep classification with respiration rates. In 2007 6th International Special Topic Conference on Information Technology Applications in Biomedicine (pp. 194-197). IEEE. doi:10.1109/ITAB.2007.4407379

Cole, R. J., Kripke, D. F., Gruen, W., Mullaney, D. J., \& Gillin, J. C. (1992). Automatic sleep/wake identification from wrist activity. Sleep, 15(5), 461-469. doi:10.1093/sleep/15.5.461 PMID:1455130

Dutta, P. (2017). Decision making in medical diagnosis via distance measures on interval valued fuzzy sets. International Journal of System Dynamics Applications, 6(4), 63-83. doi:10.4018/IJSDA.2017100104

Elfouly, F. H., Ramadan, R. A., Mahmoud, M. I., \& Dessouky, M. I. (2017). Efficient data reporting in a multi-object tracking using WSNs. International Journal of System Dynamics Applications, 6(1), 38-57. doi:10.4018/IJSDA.2017010103

Eyal, S., \& Baharav, A. (2017, September). Sleep insights from the finger tip: How photoplethysmography can help quantify sleep. In 2017 Computing in Cardiology (CinC) (pp. 1-4). IEEE.

Galland, B. C., Kennedy, G. J., Mitchell, E. A., \& Taylor, B. J. (2012). Algorithms for using an activity-based accelerometer for identification of infant sleep-wake states during nap studies. Sleep Medicine, 13(6), 743-751. doi:10.1016/j.sleep.2012.01.018 PMID:22542788

Hong, P., Chengde, L., Linkai, L., \& Qifeng, Z. (2007, May). Accuracy of classifier combining based on majority voting. In 2007 IEEE International Conference on Control and Automation (pp. 2654-2658). IEEE. doi:10.1109/ICCA.2007.4376843

Kwon, S., Park, D., Bang, H., Park, J., \& Park, Y. (2014, January). Semantic sleep management service in healthcare sensor networks. In 2014 IEEE International Conference on Consumer Electronics (ICCE) (pp. 268-269). IEEE. doi:10.1109/ICCE.2014.6775999

Lu, X., Liu, Z., \& He, J. (2016, August). Maximum likelihood approach for low-cost MEMS triaxial accelerometer calibration. In 2016 8th International Conference on Intelligent Human-Machine Systems and Cybernetics (IHMSC) (Vol. 1, pp. 179-182). IEEE. 
Majhi, S. K. (2018). An efficient feed foreword network model with sine cosine algorithm for breast cancer classification. International Journal of System Dynamics Applications, 7(2), 1-14. doi:10.4018/IJSDA.2018040101

Netti, K., \& Radhika, Y. (2015, December). A novel method for minimizing loss of accuracy in Naive Bayes classifier. In 2015 IEEE International Conference on Computational Intelligence and Computing Research (ICCIC) (pp. 1-4). IEEE. doi:10.1109/ICCIC.2015.7435801

Panda, M. (2019). Software Defect Prediction Using Hybrid Distribution Base Balance Instance Selection and Radial Basis Function Classifier. International Journal of System Dynamics Applications, 8(3), 53-75. doi:10.4018/IJSDA.2019070103

Ramadan, R. A., \& Altamimi, A. B. (2017). Hierarchal Fuzzy Logic Controller and Internet of Things (IoT) Information: Disease Spreading as a Test Case. International Journal of System Dynamics Applications, 6(3), 59-86. doi:10.4018/IJSDA.2017070104

Sadeh, A., Sharkey, M., \& Carskadon, M. A. (1994). Activity-based sleep-wake identification: An empirical test of methodological issues. Sleep, 17(3), 201-207. doi:10.1093/sleep/17.3.201 PMID:7939118

Sanders, T. H., McCurry, M., \& Clements, M. A. (2014, August). Sleep stage classification with cross frequency coupling. In 2014 36th Annual International Conference of the IEEE Engineering in Medicine and Biology Society (pp. 4579-4582). IEEE. doi:10.1109/EMBC.2014.6944643

Sano, A., \& Picard, R. W. (2014, August). Comparison of sleep-wake classification using electroencephalogram and wrist-worn multi-modal sensor data. In 2014 36th Annual International Conference of the IEEE Engineering in Medicine and Biology Society (pp. 930-933). IEEE.

Sathyanarayana, A., Joty, S., Fernandez-Luque, L., Ofli, F., Srivastava, J., Elmagarmid, A., Arora, T., \& Taheri, S. (2016). Sleep quality prediction from wearable data using deep learning. JMIR mHealth and uHealth, 4(4), e125. doi:10.2196/mhealth.6562 PMID:27815231

Sathyanarayana, A., Joty, S., Fernandez-Luque, L., Ofli, F., Srivastava, J., Elmagarmid, A., Arora, T., \& Taheri, S. (2016). Sleep quality prediction from wearable data using deep learning. JMIR mHealth and uHealth, 4(4), e125. doi:10.2196/mhealth.6562 PMID:27815231

Singh, P. K., Sarkar, R., \& Nasipuri, M. (2015). Word-level script identification using texture based features. International Journal of System Dynamics Applications, 4(2), 74-94. doi:10.4018/ijsda.2015040105

Suhas, S. R., Vijendra, S., Burk, J. R., Lucas, E. A., \& Behbehani, K. (2006). Classification of Cheyne Stokes breathing and obstructive sleep apnea using ECG. In 2006 International Conference of the IEEE Engineering in Medicine and Biology Society (pp. 3561-3564). IEEE. doi:10.1109/IEMBS.2006.259983

Tataraidze, A., Anishchenko, L., Korostovtseva, L., Kooij, B. J., Bochkarev, M., \& Sviryaev, Y. (2015, August). Sleep stage classification based on respiratory signal. In 2015 37th Annual International Conference of the IEEE Engineering in Medicine and Biology Society (EMBC) (pp. 358-361). IEEE. doi:10.1109/EMBC.2015.7318373

Tataraidze, A., Korostovtseva, L., Anishchenko, L., Bochkarev, M., Sviryaev, Y., \& Ivashov, S. (2016, August). Bioradiolocation-based sleep stage classification. In 2016 38th Annual International Conference of the IEEE Engineering in Medicine and Biology Society (EMBC) (pp. 2839-2842). IEEE. doi:10.1109/EMBC.2016.7591321

Xiaoying, X., \& Sheng, F. (2012, June). A synthesized sampling approach for improving the prediction of imbalanced classification. In 2012 IEEE International Conference on Computer Science and Automation Engineering (pp. 615-619). IEEE. doi:10.1109/ICSESS.2012.6269542

Yeo, M., Koo, Y. S., \& Park, C. (2017). Automatic detection of sleep stages based on accelerometer signals from a wristband. IEIE Transactions on Smart Processing \& Computing, 6(1), 21-26. doi:10.5573/IEIESPC.2017.6.1.021

Yimman, S., Praesomboon, S., Klunium, S., Navarattara, S., \& Dejhan, K. (2005, December). Two-Pole Bandpass Filter Based on All-pass Filter. In 2005 5th International Conference on Information Communications \& Signal Processing (pp. 569-573). IEEE. doi:10.1109/ICICS.2005.1689111

Zhang, L., Xiong, J., Zhao, H., Hong, H., Zhu, X., \& Li, C. (2017, May). Sleep stages classification by CW Doppler radar using bagged trees algorithm. In 2017 IEEE Radar Conference (RadarConf) (pp. 788-791). IEEE. doi:10.1109/RADAR.2017.7944310 
Sabyasachi Chakraborty is pursuing his Master's in computer engineering from Inje University. He has previously worked on multiple real-life projects related to computer vision, data sciences, biosignal analysis and text mining. His research interest aligns with Computer Vision, Artificial Intelligence, BioSignal Processing, Algorithms, and Blockchain.

Satyabrata Aich is working as a research professor in the field of computer engineering. He has published many research papers in journals and conferences in the realms of Machine learning, Text mining, and Supply Chain Management. His research interests are natural language processing, Machine learning, supply chain management, Bioinformatics, Artificial Intelligence,Blockchain Hee-Cheol Kim received his BSc at the Department of Mathematics, MSc at the Department of Computer Science in SoGang University in Korea, and Ph.D. at Numerical Analysis and Computing Science, Stockholm University in Sweden in 2001. He is a Professor at the Department of Computer Engineering and Head of the Institute. Digital Anti-aging Healthcare, Inje University in Korea. His research interests include Machine learning, Text mining, Bio Informatics. 